\title{
Nonlinear effects of phonon fluctuations on transport through nanoscale junctions
}

\author{
D. F. Urban, ${ }^{1,2, *}$ R. Avriller, ${ }^{2}$ and A. Levy Yeyati ${ }^{2}$ \\ ${ }^{1}$ Physikalisches Institut, Albert-Ludwigs-Universität, 79104 Freiburg, Germany \\ ${ }^{2}$ Departamento de Física de la Materia Condensada C-XII, Facultad de Ciencias, \\ Universidad Autónoma de Madrid, E-28049, Madrid, Spain
}

(Dated: June 22, 2018)

\begin{abstract}
We analyze the effect of electron-phonon coupling on the full counting statistics of a molecular junction beyond the lowest order perturbation theory. Our approach allows to take into account analytically the feedback between the non-equilibrium phonon and electronic distributions in the quantum regime. We show that for junctions with high transmission and relatively weak electron-phonon coupling this feedback gives rise to increasingly higher nonlinearities in the voltage dependence of the cumulants of the transmitted charges distribution.

PACS numbers: 73.63.Rt, 73.23.-b, 73.63.-b, 72.70.+m
\end{abstract}

Single molecule junctions and atomic chains suspended between metallic electrodes constitute a fascinating playground to explore the interplay between electronic and vibronic degrees of freedom, see e.g. Refs. 1 $\left[\begin{array}{l}5 \\ \text { ] }\end{array}\right.$ interest is now not only restricted to the understanding of the mean current-voltage characteristics but has been extended to noise properties $\underline{\underline{6}}$ and, more generally, to the full counting statistics (FCS) of the transmitted charges $\stackrel{7.8}{7}$ An intense theoretical activity has been focussed on the analysis of the simplest model consisting in a resonant level coupled to a single phonon mode in the quantum coherent regime $\stackrel{\underline{7-11}}{=}$ So far, however, several aspects of this problem remain to be clarified. A serious limitation of existing transport theories is that they do not take into account the influence of the non-equilibrium phonon fluctuations in the statistics of the transmitted electrons, namely the feedback of the phonon dynamics on the current-noise properties $\frac{12}{2}$ This limitation is associated with the breakdown of perturbation theory beyond the lowest order in electron-phonon (e-ph) coupling as reported in Refs. 13]. These works, which demonstrate the necessity of including non-perturbative effects in the analysis, are, however, limited to equilibrium properties of bulk materials or to individual molecules in the sequential tunneling regime.

In this work we demonstrate, by a partial resummation of the perturbative expansion, the great impact of the feedback of the phonon dynamics on the quantum transport properties through nanoscale junctions with high transmission and relatively weak e-ph coupling. The actual signatures of phonon fluctuations result from the interplay of several energy scales, i.e. the tunneling rate $\Gamma$ of electrons, the e-ph coupling $\lambda$, the phonon frequency $\omega_{0}$ and the relaxation rate $\propto \eta$ of the local phonon mode due to the coupling with bulk phonons. Depending on the specific sample considered and the efficiency of the relaxation mechanism for the phonon population, one might obtain a regime characterized by a thermal phonon population when $\eta \gg \lambda^{2} \omega_{0} / \Gamma^{2}$ (equilibrated phonons) or a regime where a strong non-equilibrium population is generated when $\eta \ll \lambda^{2} \omega_{0} / \Gamma^{2}$ (unequilibrated phonons). We analyze the crossover between the two regimes and demonstrate that in the regime of unequilibrated phonons the electronic current-noise shows a strong nonlinear behavior as a function of the applied voltage for $V>\omega_{0}$. We attribute these nonlinearities to a dynamical feedback of electronic quantum fluctuations that strongly renormalize the parameters describing the local phonon mode. This mechanism affects also the higher-order cumulants $C_{n}$ of the current operator which exhibit growing nonlinearities with the order of the considered cumulant. In particular we find the scaling

$$
\delta C_{n+1} / \delta C_{n} \sim V / \omega_{0}
$$

for the $\lambda$-dependent part $\delta C_{n}$ of the cumulants which is valid for $\Gamma \gg V \gg \omega_{0} \gg \eta$. Despite the experimentally weak e-ph coupling these strong nonlinearities become sizable in the regime corresponding to recent experiments on transport through small molecules using mechanically controllable break junctions. $\frac{1}{n}$ Therefore they are expected to be essential to correctly capture the noise properties above the inelastic threshold.

For our study, we use the model sketched in Fig. 1(a) corresponding to a Hamiltonian

$$
H=\Delta d^{\dagger} d+\omega_{0} a^{\dagger} a+H_{L}+H_{R}+V_{T}+V_{e-p h} .
$$

Here $d$ is the annihilation operator for the electron on the dot level at energy $\Delta$ and $a$ is the annihilation operator for a phonon of energy $\omega_{0}$ (we use $e=\hbar=1$ ). The left and right electrodes, described by $H_{L / R}$, are modeled as noninteracting fermionic continua with respective field operators $\Psi_{L / R}(x)$ and the corresponding electronic density of states $\rho_{0}$ is assumed to be energy independent. The electrodes are held at different chemical potentials giving rise to a voltage drop $V>0$. Electrons are allowed to tunnel between the electrodes and the dot, described by the local tunnel Hamiltonian $V_{T}=d^{\dagger}\left(\gamma_{L} \Psi_{L}(0)+\gamma_{R} \Psi_{R}(0)\right)+$ h.c. with left(right) hopping amplitude $\gamma_{L(R)}$. Finally, the e-ph interaction is given in terms of the displacement operator $\Phi=a+a^{\dagger}$ by $V_{e-p h}=\lambda \Phi d^{\dagger} d$. In the present work we concentrate on the regime $\Gamma_{L, R}=\pi \rho_{0} \gamma_{L, R}^{2} \gg V, \omega_{0}, \Delta, \lambda$ which is relevant for experiments involving small molecules $\frac{1}{}$ and chains of atoms $\stackrel{2}{2}$ The analysis will mainly address the case of perfect transmission $\left(\Gamma_{L}=\Gamma_{R}=\Gamma / 2\right.$ and $\left.\Delta \sim 0\right)$ where the feedback mechanism can be explained in terms of simple analytical expressions. 
(a)

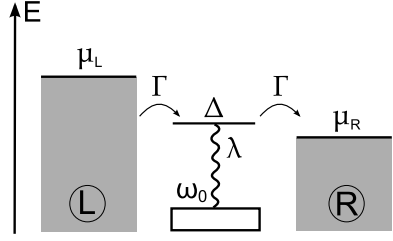

(d)

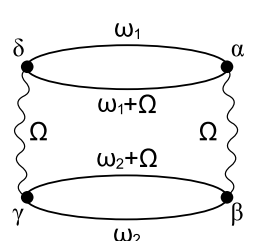

(e)

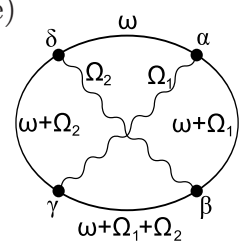

(b)

(c)

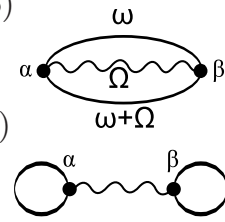

(f)

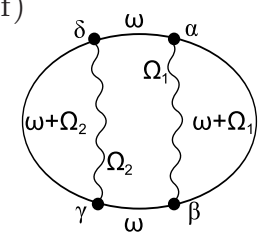

FIG. 1: (a) Sketch of the model system. (b) Exchange(Fock) and (c) Hartree diagram, both appearing in the linked cluster expansion of the CGF at order $\lambda^{2}$. Bottom: fourth order diagrams, i.e. (d) double-bubble, (e) double-exchange, and (f) rainbow diagram.

The FCS of charge transport through the dot is derived by calculating the cumulant generating function (CGF) $\chi(\xi)=\chi_{0}(\xi)+\delta \chi(\xi)$ of the corresponding probability distribution $P(Q)$ to transfer the charge $Q$ during the measuring time $t_{0}$. The CGF of the non-interacting system $\chi_{0}(\xi)$ is given by the Levitov-Lesovik result $\stackrel{14,15}{1}$ The correction due to finite e-ph coupling is given by

$$
\delta \chi(\xi)=-\ln \left\langle T_{\mathcal{C}} \exp \left(-i \int_{\mathcal{C}} d t V_{e-p h}(t) d t\right)\right\rangle_{0} .
$$

Here the expectation value is taken with respect to the non-interacting Hamiltonian $H_{0}=H-V_{e-p h}$ and integration runs over the Keldysh contour $\mathcal{C}$ with time ordering $T_{\mathcal{C}}$ along $\mathcal{C}$. The counting field $\xi(t)$ is included in the tunnel Hamiltonian $V_{T}$ through the substitution $\tilde{\gamma}_{L}=\gamma_{L} e^{-i \xi(t) / 2}$, taking the value $+(-) \xi$ on the forward(backward) branch of $\mathcal{C}$. The CGF allows to access the cumulants $C_{n}$ of $P(Q)$ by successive derivation with respect to the counting field, $C_{n}=\left.\frac{(-i)^{n}}{t_{0}} \frac{\partial^{n} \chi}{\partial \xi^{n}}\right|_{\xi=0}$.

The linked cluster expansion of $\delta \chi$ in the e-ph coupling $\lambda$ can be evaluated in terms of the noninteracting electron Green function $(\mathrm{GF}) G_{0}\left(t, t^{\prime}\right)=-i\left\langle T_{\mathcal{C}} d(t) d^{\dagger}\left(t^{\prime}\right)\right\rangle_{0}$ and phonon propagator $D_{0}\left(t, t^{\prime}\right)=-i\left\langle T_{\mathcal{C}} \Phi(t) \Phi\left(t^{\prime}\right)\right\rangle_{0}$. The Keldysh components $(\alpha, \beta= \pm)$ of the latter read

$$
D_{0}^{\alpha \beta}(\omega)=\frac{1}{\omega-\alpha \omega_{0}+i \eta}-\frac{1}{\omega+\beta \omega_{0}-i \eta},
$$

which includes the finite phenomenological parameter $\eta$ describing the coupling with bulk phonons. The first term contributing to the expansion of $\delta \chi$ is $\propto \lambda^{2}$ and involves the calculation of a Hartree-type and an exchange(Fock)-type diagram, c.f. Fig. 1(b)-(c). The latter term was recently discussed in detail ${ }^{7,8}$ and was shown to be responsible for the jump in the derivative with respect to voltage of the cumulants near the inelastic threshold at $V=\omega_{0}$.
On the other hand, the Hartree diagram leads to a shift of the dot level position $\Delta$ and this renormalization is almost independent of voltage. This statement is found to hold also for the Hartree-type diagrams of higher order in $\lambda$ within the parameter range discussed in this article.

The next order term $\lambda^{4} \delta \chi_{4}(\xi)$ involves the evaluation of three diagrams, which are shown in the lower panel of Fig. 1. The nomenclature double bubble (DB), double exchange (DX) and rainbow (RB) refers to the Feynman graphs of the corresponding self-energies. In the limit of $\Gamma \gg V, \omega_{0}$ and at zero temperature, we can give analytical expressions for the DX and RB diagrams and find that they are of order $\lambda^{4} / \Gamma^{4}$. They can thus be safely neglected in comparison to the dominant DB diagram $\delta \chi_{4}^{(D B)}(\xi) \propto \lambda^{4} /\left(\Gamma^{2} \eta^{2}\right)$ which diverges in the limit of vanishing external damping $\eta$.

With the corresponding diagrams of higher order in $\lambda$ all being found to be divergent, we suggest a resummation of this subset of diagrams. The Dyson equation $\hat{D}=\hat{D}_{0}+\hat{D}_{0} \hat{\Sigma}_{p h} \hat{D}$ for this RPA-type dressing of the phonon GF involves the $\xi$ - and $V$-dependent phonon selfenergy

$$
\hat{\Sigma}_{p h}(\omega)=-i \lambda^{2}\left(\begin{array}{cc}
\Pi^{++}(\omega) & -\Pi^{+-}(\omega) \\
-\Pi^{-+}(\omega) & \Pi^{--}(\omega)
\end{array}\right),
$$

where $\hat{\Pi}(\omega)$ is the charge polarization loop,

$$
\Pi^{\alpha \beta}(\omega)=\int \frac{d \varepsilon}{2 \pi} G_{0}^{\alpha \beta}(\omega+\varepsilon) G_{0}^{\beta \alpha}(\varepsilon) .
$$

Note that in the parameter range discussed in the following, i.e. $\lambda^{2} / \omega_{0} \Gamma \leq 0.25$, we found the RPA approximation to be charge conserving up to the chosen numerical accuracy. The main result of the resummation is a shift and a broadening of the phonon mode by adding a finite lifetime due to the coupling to the electrons. A crucial aspect is to realize that this mode renormalization is $\xi$ dependent, i.e. it is sensitive to the counting-field of the tunneling electrons. This has important consequences as discussed below. In the limit $\Gamma \gg V, \omega$ we can expand

$$
\begin{aligned}
& \Pi^{-+}(\omega) \simeq \frac{1}{\pi \Gamma^{2}}\left\{\omega \theta(\omega)-\sum_{\sigma= \pm} e^{i \sigma \xi} \frac{\sigma V-\omega}{2} \theta(\omega-\sigma V)\right\} \\
& \Pi^{\alpha \alpha}(\omega) \simeq \frac{\alpha}{i \pi \Gamma}+\frac{2|\omega|+(V-|\omega|) \theta(V-|\omega|)}{2 \pi \Gamma^{2}}
\end{aligned}
$$

and thereby find four poles $\Omega_{i}$ of $D^{\alpha \beta}(\omega)$. For $V>\omega_{0}$, the two poles having a positive real part read

$$
\begin{gathered}
\Omega_{1 / 2}=\left(\omega_{0} \pm i \eta\right)-\frac{\lambda^{2}}{\pi \Gamma}-\frac{\lambda^{4}}{2 \pi^{2} \Gamma^{2} \omega_{0}} \\
\pm \frac{i \lambda^{2}}{2 \pi \Gamma^{2}}\left[e^{-i \xi}\left(V-\omega_{0} \mp i \eta\right)-\left(V+\omega_{0} \pm i \eta\right)\right]+\mathcal{O}\left(\Gamma^{-3}\right)
\end{gathered}
$$

and due to symmetry $\Omega_{3 / 4}=-\Omega_{1 / 2}$.

This phonon renormalization goes beyond the widely used approximation of the non-equilibrium phonon population based on a rate equation (RE) description $3.4,8$ 

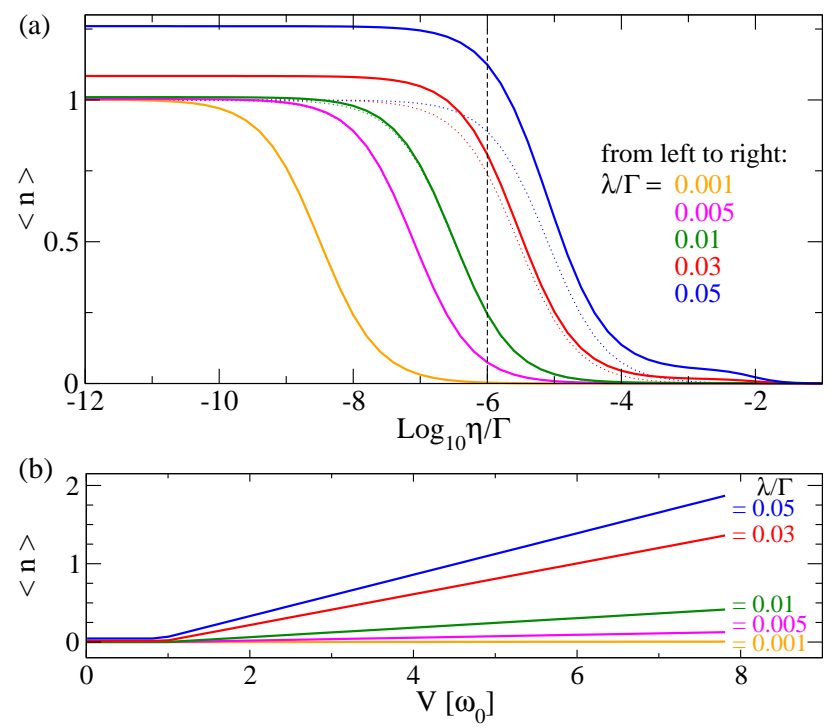

FIG. 2: (a) Mean number of phonons $\langle n\rangle$ at $V=5 \omega_{0}$ and $\omega_{0} / \Gamma=0.01$ as a function of $\eta$ shown for various values of $\lambda$. For comparison, the dotted curves show the results of the rateequation approach, Eq. (10), for the same parameters. (b) Voltage dependence of $\langle n\rangle$ for $\eta / \Gamma=10^{-6}$ and $\omega_{0} / \Gamma=0.01$.

Within this RE approximation, the mean number of phonons $\langle n\rangle$ at zero temperature is given by $\underline{\underline{4}}$

$$
\langle n\rangle_{R E}=\frac{\lambda^{2} \omega_{0}}{4 \pi \eta \Gamma^{2}+4 \lambda^{2} \omega_{0}}\left(\frac{|V|}{\omega_{0}}-1\right) \theta\left(|V|-\omega_{0}\right),
$$

which qualitatively describes the crossover between the equilibrated and unequilibrated phonon regimes (for $\eta \gg$ $\lambda^{2} \omega_{0} / \Gamma^{2}$ and $\eta \ll \lambda^{2} \omega_{0} / \Gamma^{2}$, respectively). In Fig. 2(a) we compare this prediction (dotted lines) with the results for $\langle n\rangle$ obtained within the renormalized perturbation theory (solid lines) for $\omega_{0}=0.01 \Gamma$ and different $\lambda$ values. Therefore we compute $\langle n\rangle$ by integrating the non-diagonal component of the dressed phonon propagator, $\langle n\rangle=-\frac{1}{2}+\left.\frac{i}{4 \pi} \int d \omega D^{+-}(\omega)\right|_{\xi=0}$. While there is a good agreement of the RE approach with our calculations when $\lambda<\omega_{0}$, discrepancies arise when $\lambda>\omega_{0}$, especially in the regime of unequilibrated phonons. The RE approach then underestimates the phonon population at a given voltage due to the neglect of the mode renormalization effects.

We can now turn to the calculation of transport properties. We evaluate $\delta \chi(\xi)$ using the dressed phonon GF,

$$
\delta \chi(\xi) \simeq \frac{t_{0} \lambda^{2}}{2 i} \sum_{\alpha, \beta= \pm}(\alpha \beta) \int \frac{d \omega}{2 \pi} \Pi^{\alpha \beta}(\omega ; \xi) D^{\beta \alpha}(\omega ; \xi)
$$

and from this deduce the current-voltage characteristics $I(V)$ as well as the current noise power (second cumulant $C_{2}$ ). Figure 3 shows representative plots for the $\lambda$-dependent contributions $\delta G$ and $\delta S^{\prime}$ to the differential conductance $G=d I / d V$ and derivative of the current noise with respect to voltage $S^{\prime}=d C_{2} / d V$, respectively, in the crossover regime $\eta \sim \lambda^{2} \omega_{0} / \Gamma^{2}$. The obtained
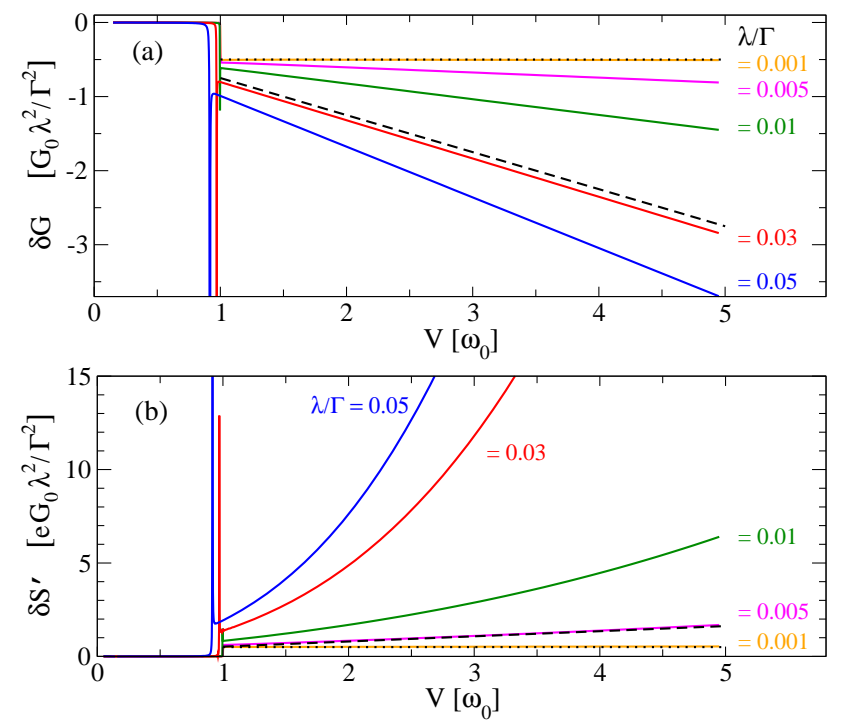

FIG. 3: (a) Differential conductance and (b) derivative of the current noise with respect to voltage as function of voltage for various values of $\lambda$. Parameters used are $\omega_{0} / \Gamma=0.01$ and $\eta / \Gamma=10^{-6}$. The dotted (dashed) lines show the results of the rate equation approach for thermally equilibrated (unequilibrated) phonons for comparison, taken from Ref. [8].

curves change dramatically with the e-ph coupling. The conductance (Fig. Ba ) shows a negative jump at $V=\omega_{0}$ discussed in detail in recent works $\mathrm{s}^{-\underline{9}}$ which is related to enhanced inelastic backscattering processes. Note that the rounded spikes at the threshold are reminiscent of the log-singularities obtained without the resummation which are pronounced only for the largest $\lambda$ values shown ${ }^{16}$ For voltages above the inelastic threshold, $\delta G(V)$ is almost linear and exhibits a crossover from a flat voltage-independent regime at low $\lambda / \Gamma=0.001$ (equilibrated phonons) to a negative slope at larger $\lambda / \Gamma=0.05$ (unequilibrated phonons). The corresponding limiting cases are plotted (dotted and dashed lines, respectively) using the RE approach of Ref. [8] and the shape of the $\delta G(V)$ curves is well reproduced by this approximation. The linear voltage dependence is directly related to the linear population of the phonon mode for $V>\omega_{0}$, c.f. Fig. 2(b), and the negative slope is due to activated absorption processes resulting from the increasing population of the local vibrational mode.

The derivative of the current noise with respect to voltage (Fig. 3b) exhibits a positive jump at $V=\omega_{0}$ as a result of the same inelastic backscattering mechanism responsible for the jump in $\delta G(V)$. However, for $V>\omega_{0}$ the behavior of the curves is qualitatively different. In the regime of equilibrated phonons, $\delta S^{\prime}(V)$ is almost voltageindependent and well reproduced by the $\mathrm{RE}$ approach. For unequilibrated phonons however, the current-noise curves develop strong nonlinearities which are absent in the RE approach. We stress that already for quite small $\lambda / \Gamma=0.005$ (corresponding to an almost thermally equilibrated regime, c.f. Fig. 22 ) the amplitude of the nonlinearities is large enough to match the (maximal) results 
of the RE approach for unequilibrated phonons (dashed line in Fig. 3b). This qualitative different behavior can be understood as follows. Within the RE approach and in the limit $\Gamma \gg \omega_{0}, V, \lambda$ in which we are interested, the correction to the FCS due to e-ph coupling is purely Poissonian, 7.8 , i.e. $\delta C_{n, R E} \propto \delta C_{1, R E}$. On the other hand, the correction to the FCS in the renormalized theory departs from being Poissonian due to the dynamical renormalization of the phonon mode. In the particular regime of unequilibrated phonons $\left(\eta \ll \lambda^{2} \omega / \Gamma^{2}\right)$ and for $\Gamma \gg V>\omega_{0}$, the dressed phonon propagator can be approximated by

$$
\hat{D} \simeq\left(\prod_{j=1}^{4} \frac{\sqrt{2 \omega_{0}}}{\omega-\Omega_{j}}\right)\left(\begin{array}{cc}
f-i \lambda^{2} \Pi^{--} & -i \lambda^{2} \Pi^{+-} \\
-i \lambda^{2} \Pi^{-+} & -f-i \lambda^{2} \Pi^{++}
\end{array}\right)
$$

with $f=\left(\omega^{2}-\omega_{0}^{2}\right) / 2 \omega_{0}$ and the poles $\Omega_{j}$ given in Eq. (9). The integral of Eq. (11) is then approximated by

$$
\begin{aligned}
\delta \chi(\xi) \simeq & \frac{t_{0} \lambda^{2}}{2 i} \sum_{j=1}^{4} \frac{4 \omega_{0}^{2}}{\prod_{k \neq j}\left(\Omega_{j}-\Omega_{k}\right)} \sum_{\alpha, \beta= \pm}(\alpha \beta) \\
& \int \frac{d \omega}{2 \pi} \frac{\Pi^{\alpha \beta}(\omega)\left[\alpha f(\omega) \delta_{\alpha \beta}-i \lambda^{2} \Pi^{-\alpha,-\beta}(\omega)\right]}{\omega-\Omega_{j}},
\end{aligned}
$$

where the prefactor can be expressed as

$$
\frac{4 \omega_{0}^{2}}{\prod_{k \neq j}\left(\Omega_{j}-\Omega_{k}\right)} \simeq \sigma_{j} \frac{i \pi \Gamma^{2}}{\lambda^{2} \omega_{0}} \frac{e^{i \xi}}{1+e^{i \xi}} \sum_{n=0}^{\infty}\left[-i \tan (\xi / 2) \frac{V}{\omega_{0}}\right]^{n}
$$

with $\sigma_{j}=-\operatorname{sign}\left(\operatorname{Im}\left(\Omega_{j}\right)\right)$. This factor therefore is identified as the source of the increasing nonlinear voltage dependence when taking successive derivatives of $\delta \chi$ with respect to $\xi$ at $\xi=0$. Finally, the most nonlinear contribution to the $\delta C_{n}$ in this regime reads

$$
\delta C_{n} \sim(-1)^{n}(n !) \frac{2 \lambda^{2} \omega_{0}}{\pi \Gamma^{2}}\left(\frac{V}{2 \omega_{0}}\right)^{n+1}
$$

which demonstrates the scaling law anticipated in the introduction. 17

In conclusion, we have analyzed the effect of e-ph interaction on the FCS of a molecular junction beyond the lowest order perturbation theory. A RPA-type resummation of diverging diagrams results in a renormalization of the phonon parameters which depends on the electron counting-field, thereby giving rise to an increasingly higher nonlinear voltage dependence in the cumulants of the transmitted charges distribution. These strong nonlinear effects are expected to be accessible in low-temperature transport experiments performed on highly conductive molecular junctions 1 and atomic chains, $\stackrel{2}{=}$ where typically the energy of the strongly coupled vibrational mode and the e-ph coupling strength are of order $\omega_{0} / \Gamma \approx 0.01$ and $\lambda / \Gamma \approx 0.1-0.01$, respectively.

The authors would like to thank F. Haupt, A. Komnik, R. Egger, H. Grabert, Jan van Ruitenbeek, J.C. Cuevas and A. Martin-Rodero for many interesting and fruitful discussions. Financial support from the Spanish MICINN under contract NAN2007-29366E (CHENANOM) is acknowledged. D. Urban and R. Avriller contributed equally to this work.
* Electronic address: urban@physik.uni-freiburg.de

1 R.H. Smit, Y. Noat, C. Untiedt, N.D. Lang, M.C. van Hemert, and J.M. van Ruitenbeek, Nature (London) 419, 906 (2002); O. Tal, M. Krieger, B. Leerink, and J.M. van Ruitenbeek, Phys. Rev. Lett. 100, 196804 (2008).

2 N. Agrait, C. Untiedt, G. Rubio-Bollinger and S. Vieira, Phys. Rev. Lett. 88, 216803 (2002).

3 T. Frederiksen, M. Brandbyge, N. Lorente, and A.-P. Jauho, Phys. Rev. Lett. 93, 256601 (2004); T. Frederiksen, M. Paulsson, M. Brandbyge, and A.-P. Jauho, Phys. Rev. B 75, 205413 (2007); M. Paulsson, T. Frederiksen, H. Ueba, N. Lorente, and M. Brandbyge, Phys. Rev. Lett. 100, 226604 (2008).

4 J. K. Viljas, J. C. Cuevas, F. Pauly, and M. Hafner, Phys. Rev. B 72, 245415 (2005);

5 L. de la Vega, A. Martin-Rodero, N. Agrait, and A. Levy Yeyati, Phys. Rev. B. 73, 075428 (2006);

6 J.-X. Zhu and A.V. Balatsky, Phys. Rev. B. 67, 165326 (2003); M. Galperin, A. Nitzan, and M.A. Ratner, Phys. Rev. B. 74, 075326 (2006).

7 T. L. Schmidt and A. Komnik, Phys. Rev. B. 80, 041307(R) (2009); R. Avriller and A. Levy Yeyati, Phys. Rev. B. 80, 041309(R) (2009).

8 F. Haupt, T. Novotny, and W. Belzig, Phys. Rev. Lett. 103, 136601 (2009).

9 R. Egger and A.O. Gogolin, Phys. Rev. B. 77, 113405
(2008)

10 A. Mitra, I. Aleiner, and A. J. Millis, Phys. Rev. B 69, 245302 (2004); M. Galperin. M. A. Ratner and A. Nitzan, J. Chem. Phys. 121, 11965 (2004); O. Entin-Wohlman, Y. Imry, and A. Aharony, Phys. Rev. B 80, 035417 (2009); O. Entin-Wohlman, Y. Imry, A. Aharony, Phys. Rev. B 81, 113408 (2010).

11 L. K. Dash, H. Ness, R. W. Godby, J. Chem. Phys. 132, 104113 (2010).

12 This feedback was recently considered in an approximate way for the computation of the mean number of phonons and the mean current (Ref. 4 and Mitra et al in [10)], but not for the current-noise nor the FCS.

13 J. P. Hague and N. d'Ambrumenil, J. Low Temp. Phys., 151, 1149-1163, (2008); A. S. Alexandrov, Europhys. Lett., 56, 92 (2001); J. Koch, M. Semmelhack, F. von Oppen and A. Nitzan, Phys. Rev. B 73, 155306 (2006).

14 L.S. Levitov, H.-W. Lee, G.B. Lesovik, J. Math. Phys. 37, 4845 (1996).

15 A.O. Gogolin and A. Komnik, Phys. Rev. B. 73, 195301 (2006).

16 Such spikes are smeared further at finite temperatures but the overall behavior of $\delta G$ and $\delta S^{\prime}$ would not be affected provided that $T \ll \omega_{0}$.

17 Note that throughout the paper we assume $V>0$. However, $C_{n}(-V)=(-1)^{n} C_{n}(V)$. 